\title{
A ATUAÇÃO DE CAPACITAÇÕES NO CENÁRIO ACADÊMICO/PROFISSIONAL PARA OS ALUNOS DE GRADUAÇÃO DA UNIVERSIDADE FEDERAL DE CAMPINA GRANDE
}

Elias Gabriel Almeida Farias Alves -elias.alves@ee.ufcg.edu.br

Universidade Federal de Campina Grande

Rua Rodrigues Alves, 1400, Bela Vista

58428-795 - Campina Grande - Paraíba

Luiz Eugênio Nunes Araujo -luiz.araujo@ee.ufcg.edu.br

Universidade Federal de Campina Grande

Rua Frei Caneca, 379, Centro

58400-295 - Campina Grande - Paraíba

Rosmânya Marlla Fialho de Lima Fonseca - rosmanya.fonseca@ee.ufcg.edu.br

Universidade Federal de Campina Grande

Rua Silva Barbosa, 975, Monte Santo

58400-825 - Campina Grande - Paraíba

Talita do Patrocínio Dantas dos Santos - talita.santos@ee.ufcg.edu.br

Universidade Federal de Campina Grande

Rua Rodrigues Alves, 1400, Bela Vista

58428-795 - Campina Grande - Paraíba

Thalis da Costa Guedes - thalis.guedes@ee.ufcg.edu.br

Universidade Federal de Campina Grande

Rua Rodrigues Alves, 1400, Bela Vista

58428-795 - Campina Grande - Paraíba

Resumo: Sob as perspectivas educacionais, a evasão no ensino superior é considerada um problema socioeducativo, visto que gera impactos negativos nas universidades. É perceptível que a taxa de evasão está aumentando cada vez mais, sendo assim, pesquisadores vêm intensificando seus estudos em busca de melhorias nessa situação. Afinal, o mercado de trabalho necessita de profissionais, principalmente capacitados. Pensando nisso, o Capítulo Estudantil PES (Sociedade de Potência e Energia) da Universidade Federal de Campina Grande (UFCG) pertencente ao Instituto de Engenheiros Eletricistas e Eletrônicos (IEEE) atua realizando capacitações de Instalações Elétricas, Energias Renováveis, Formatação de Computadores e Photoshop. Por conseguinte, essas capacitações puderam contribuir veementemente para o engajamento, formação pessoal e profissional e o interesse para a permanência dos alunos no curso de Engenharia Elétrica. 


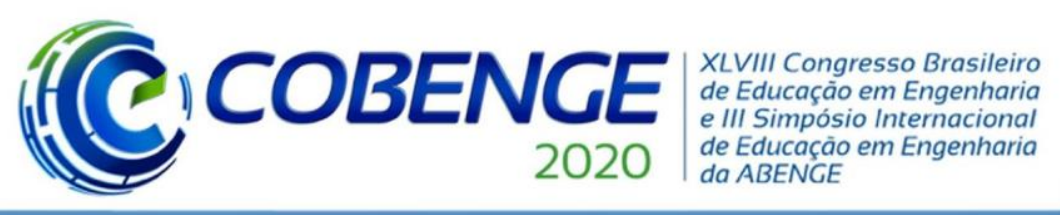

"Os desafios para formar hoje o engenheiro do amanhã"

Palavras-chave: Educação, Capacitação, Engenharia, IEEE, PES.

\section{INTRODUÇÃO}

Há uma enorme escassez de profissionais nas áreas de ciências exatas e engenharias. A principal causa disso, é a alta evasão de estudantes de cursos dessas áreas. De acordo com o Instituto Nacional de Estudos e Pesquisas Educacionais Anísio Teixeira (INEP), o Brasil forma cerca de 40 mil engenheiros por ano, enquanto na Rússia, Índia e China, formam, respectivamente, 190 mil, 220 mil e 650 mil.

Silva Filho (2007), ressalta que os motivos pelos quais os alunos iniciam, mas não concluem determinado curso, são sociais, acadêmicos e econômicos. Com base em estudos, a maior razão está na dificuldade que os alunos têm nas disciplinas do ciclo básico, tendo em vista que são mais focadas em física e matemática. Buscando melhorar essa problemática, Tontini e Walter (2014) aconselham as instituições de ensino superior reformular o currículo do curso, visando incluir atividades e disciplinas práticas no início do curso, com o intuito de motivar, interessar e identificar $\mathrm{o}$ aluno $\mathrm{em}$ sua futura profissão.

Pensando nisso, o Instituto de Engenheiros Eletricistas e Eletrônicos (IEEE) atua diretamente nesse impasse. O IEEE é uma organização mundial, que inicialmente era formada apenas por engenheiros, mas que atualmente comporta diversas áreas. Dessa forma, essas áreas são ramificadas em Sociedades formando essa organização, como por exemplo, a Sociedade de Potência e Energia (PES). Afinal, o IEEE tem como principal objetivo transportar informações sobre tecnologia para a sociedade, possibilitando oportunidades de crescimento pessoal e profissional, através de programas, projetos, atividades, conferências ou eventos. Com isso, o Capítulo Estudantil IEEE PES da Universidade Federal de Campina Grande (UFCG) oferece dessas oportunidades, isto é, apoio técnico e pessoal; atividades extracurriculares; e, em especial, capacitações para os estudantes de engenharia e/ou interessados na área.

Por conseguinte, procurando melhorar a permanência e qualificação dos alunos no curso de engenharia, os voluntários do Capítulo Estudantil IEEE PES UFCG propõem capacitações em: Photoshop, Instalações Elétricas, Formatação de Computadores e Energias Renováveis.

O público alvo são, principalmente, os alunos pouco engajados em alguma atividade extracurricular e os que estão buscando qualificação profissional. Todas as capacitações são ministradas de forma dinâmica e fácil entendimento, contendo aulas teóricas e práticas.

Além de engajar, interagir, estimular e qualificar os participantes, também oferece a oportunidade para que, em algum momento, possam ser os novos ministrantes das próximas capacitações.

\section{METODOLOGIA}

A fim de expandir o conhecimento e as habilidades de nossos voluntários para além das disciplinas abordadas na grade curricular da graduação, o Capítulo Estudantil IEEE PES UFCG considerou interessante promover capacitações internas que pudessem ajudar no desenvolvimento de competências, que são externas a vida acadêmica e extremamente 
(C) COBENCE

"Os desafios para formar hoje o engenheiro do amanhã"

necessárias para alguns trabalhos dentro no nosso grupo.

Estudamos a necessidade de cada tema abordado nas capacitações, analisando a contribuição que traria ao Capítulo e também a vida profissional dos nossos voluntários. No decorrer desta seção explicaremos como ocorreu cada uma dessas capacitações.

\subsection{Capacitação de Instalações Elétricas}

Com o intuito de gerar uma rotatividade entre os membros da gestão do Capítulo Estudantil IEEE PES UFCG, como também uma forma de integralizar ainda mais os voluntários, dandolhes a oportunidade de crescer dentro da organização, surgiu a ideia de realizar a capacitação de instalações

elétricas.

Inicialmente, durante uma reunião geral do capítulo, foi perguntado aos presentes que possuíam previamente um conhecimento técnico, se eles estariam dispostos a ministrar as próximas edições do minicurso de instalações elétricas, curso este já presente nas atividades do grupo PES. Após o recrutamento dos voluntários, se deu de fato a capacitação.

O preparo ocorreu no Laboratório de Instalações Elétricas (LIE), cedido pela universidade, sendo supervisionado por um voluntário já formado em Engenharia Elétrica, na qual já foi monitor da disciplina supracitada, além de um técnico em eletrotécnica, atual ministrante do curso, que repassaram todas as recomendações necessárias para segurança de todos. A capacitação teve como base de apoio uma apostila confeccionada especialmente para realização do curso, onde nela estava contida várias montagens referentes a parte prática de instalações.

Após a apresentação das instalações, cada voluntário na sua respectiva bancada, como mostrado na Figura 1, deu início ao processo das montagens. Ao final, é feito uma explanação de todo conteúdo visto, para que todas as dúvidas sejam sanadas.

Figura 1 - Prática da capacitação de Instalações Elétricas.

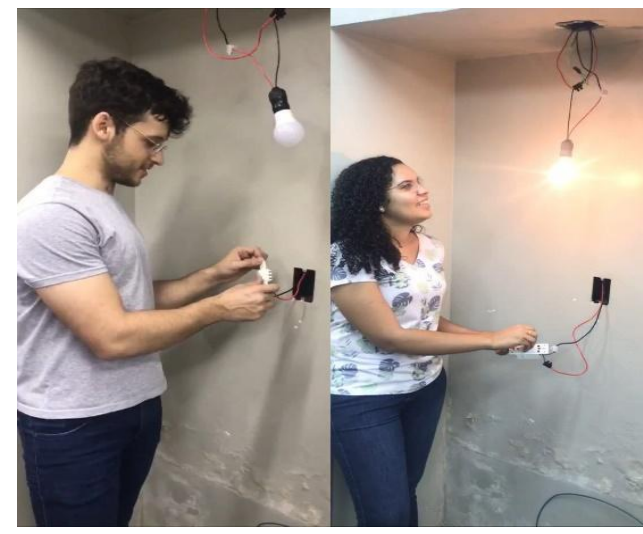

Fonte: Autoria própria.

\subsection{Capacitação de Photoshop}

A capacitação de Photoshop foi idealizada e planejada de forma a focar nos pontos mais populares, para serem repassados aos participantes, de forma prática para melhor absorção do conteúdo ministrado. Antes de qualquer apresentação teórica, o ministrante faz as devidas 
orientações para a correta instalação do software em cada computador.

Todo o assunto foi exposto por meio de uma extensão de tela, de maneira que o auxiliador demonstrasse a todos os presentes, o que deveria ser feito. O preparo da capacitação foi cuidadosamente elaborado pelo Coordenador de Marketing do Ramo Estudantil IEEE e Webmaster da PES UFCG na gestão de 2019, tendo em vista sua vasta experiência no tema, como também na organização.

A divulgação foi realizada por meio das redes sociais do Capítulo e do Ramo IEEE, bem como no grupo do e-mail institucional do Departamento de Engenharia Elétrica (DEE), onde foi repassado um link contendo todas as informações de horário, data e local, para os interessados efetuarem a inscrição. Além disso, foi questionado ao partícipe a disponibilidade de levar seu próprio computador ou não, tendo em vista que o laboratório utilizado não dispunha de um número suficiente de máquinas. Assim como todas as qualificações, essa também ocorreu dentro da universidade - com permissão da UFCG - em um dos laboratórios do curso de Ciências da Computação.

A capacitação inicia-se de fato com uma pequena explanação sobre o Capítulo PES, esclarecendo dúvidas recorrentes dos voluntários. Posteriormente, é feita as devidas recomendações para um uso saudável de todos os computadores utilizados, e em seguida, o ministrante aborda algumas práticas de Photoshop, como por exemplo: produção de artes, templates e montagens com fotos; correção e edição de fotos; e como melhorar a qualidade de imagens.

Por fim, tirou-se as dúvidas que foram surgidas no decorrer da aula e agradecemos a participação deles, dando-lhes o certificado de participação, como mostrado na Figura 3.

Figura 2 - Entrega de certificados da capacitação de Photoshop.

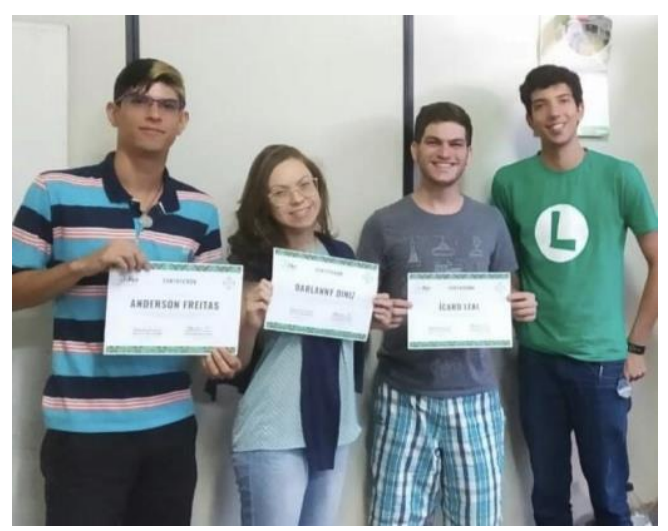

Fonte: Autoria própria.

\subsection{Capacitação de Formatação de Computador}

A ideia da capacitação de formatação de computador surgiu diante da demanda apresentada por alguns voluntários interessados em aprender como realizar o procedimento. Então, o capítulo entrou em contato com um voluntário que possuía experiência no assunto para ensinar aos interessados. 
C.COBENCE 2020 (t)

"Os desafios para formar hoje o engenheiro do amanhã"

Depois de achar um local disponível e checar a melhor data de realização, foi enviado um formulário de inscrição. Os interessados compareceram à capacitação e puderam aprender de forma teórica e também prática, todos os passos para realizar a formatação. $\mathrm{O}$ ministrante mostrou quais os programas necessários a serem baixados; os sites importantes para o processo; a importância de salvar alguns arquivos em um aparelho de armazenamento remoto ou na nuvem para evitar perda dos mesmos; demonstrou como deve ser feito o setup e a finalização do processo.

Figura 3 - Capacitação de formatação de PC.

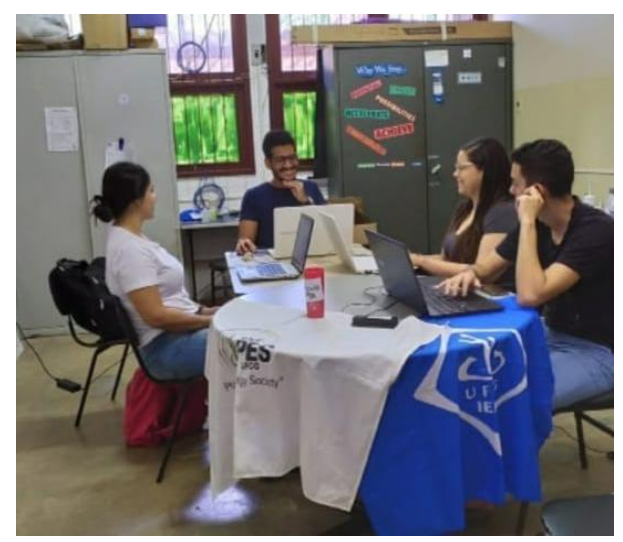

Fonte: Autoria própria.

\subsection{Capacitação RES}

O Capítulo Estudantil IEEE PES UFCG promove nas escolas da rede pública de Campina Grande a realização de um projeto, intitulado Renewable Engineering in Schools (RES), que tem por objetivo levar o ensino teórico e prático sobre energias renováveis a essas escolas parceiras. Para que tudo isso seja possível, é preciso que os voluntários estejam capacitados para tais atividades. Pensando nisso, foi criado e desenvolvido uma capacitação sobre o conteúdo e uso dos kits utilizados nas aulas.

A capacitação iniciou-se com uma breve explanação sobre o RES e logo em seguida sobres as 3 principais energias abordadas no projeto: a solar, a eólica e a eletrólise. Logo em seguida foram apresentados os kits utilizados nas intervenções e formaram-se grupos para a montagem. Depois de concluir essas etapas, foram criados grupos de estudos sobre cada uma das 3 formas de energia, com o intuito de produzir uma apostila que atualmente está em fase de finalização. 
Figura 4 - Primeira capacitação RES.

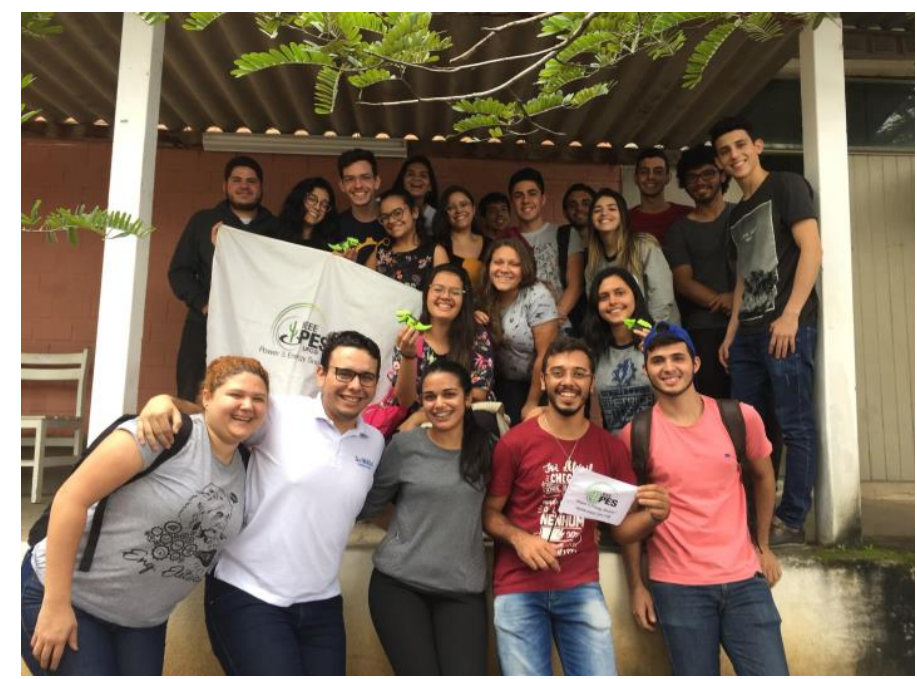

Fonte: Autoria própria.

\section{RESULTADOS}

Desde o ano de 2016 o Capítulo Estudantil IEEE PES UFCG aplica suas capacitações na Universidade Federal de Campina Grande e atinge diretamente os alunos do curso de Engenharia Elétrica. Cerca de 100 alunos já foram beneficiados com essas capacitações, o que gera resultados positivos para o desempenho tanto nas disciplinas durante a graduação, quanto em projetos de pesquisas, e além disso, serve como facilitador de problemas pessoais e técnicos.

Posto isso, ao término das capacitações são levantados dados de satisfação por meio de formulário enviados para os alunos que participaram das capacitações com o intuito de melhorar a metodologia e materiais que são utilizados, como demonstra no Gráfico 1. 
Gráfico 1 - Grau de satisfação das capacitações.

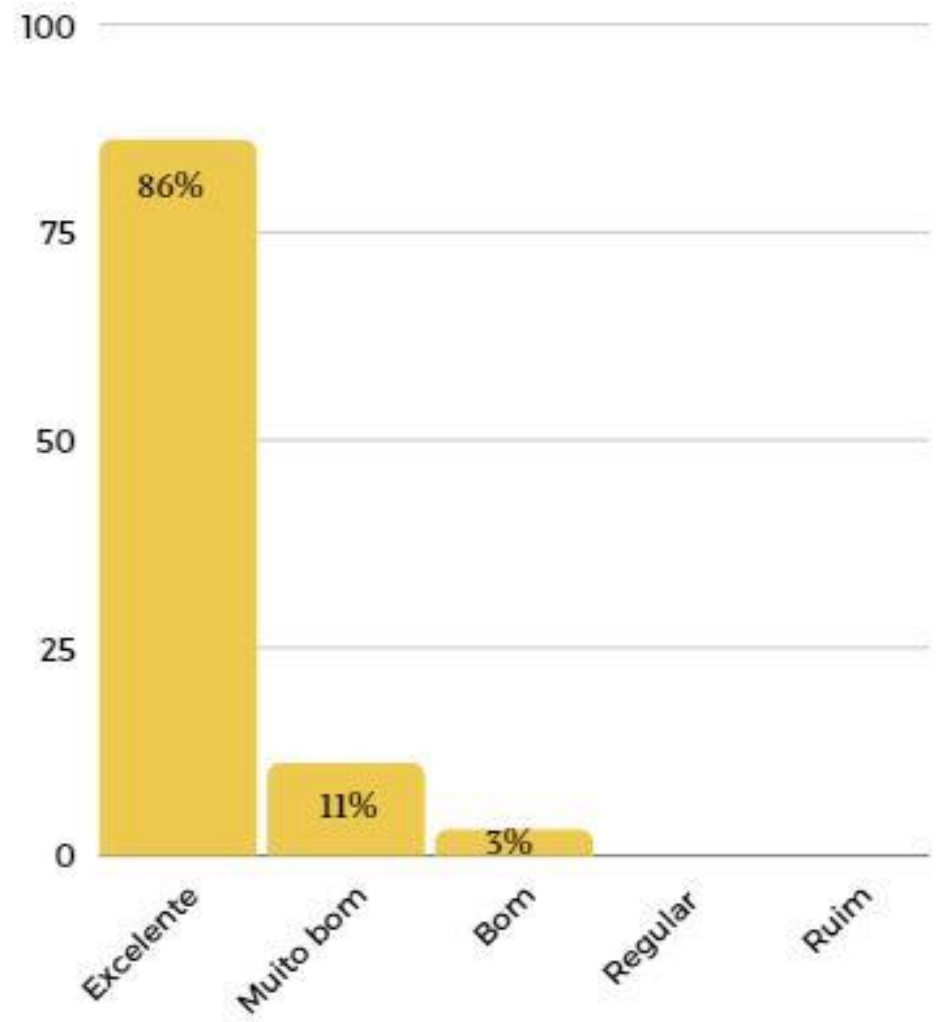

Fonte: Autoria própria.

É possível destacar que os alunos que participaram das capacitações e que realizaram a avaliação, ficaram satisfeitos, pois a maioria dos feedbacks recebidos mostrou que as capacitações estão com um bom desempenho e que possui parâmetros excelentes, como metodologia e duração.

Além disso, foi realizado mais duas perguntas para verificar se as capacitações estavam tendo aplicabilidade e para saber de forma geral se os participantes indicariam as capacitações para outras pessoas. Chegando assim a dados de que $71,4 \%$ das pessoas consideraram excelente a aplicabilidade dos conhecimentos adquiridos e $28,6 \%$ avaliaram como muito bom, como mostrado no Gráfico 2. No que se diz respeito às indicações, cerca de $85,7 \%$ indicaria as capacitações e 14,3\% optaram pela opção talvez, conforme mostrado no Gráfico 3. 
Gráfico 2 - Avaliação da aplicabilidade das capacitações.

75

50

$71,4 \%$

25

$$
28.6 \%
$$

0

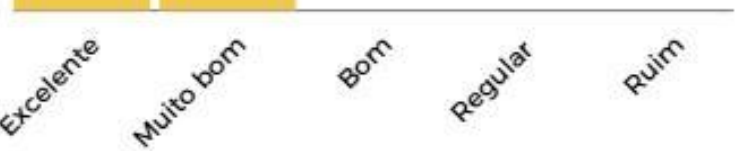

Fonte: Autoria própria.

Gráfico 3 - Avaliação indicação das capacitações.

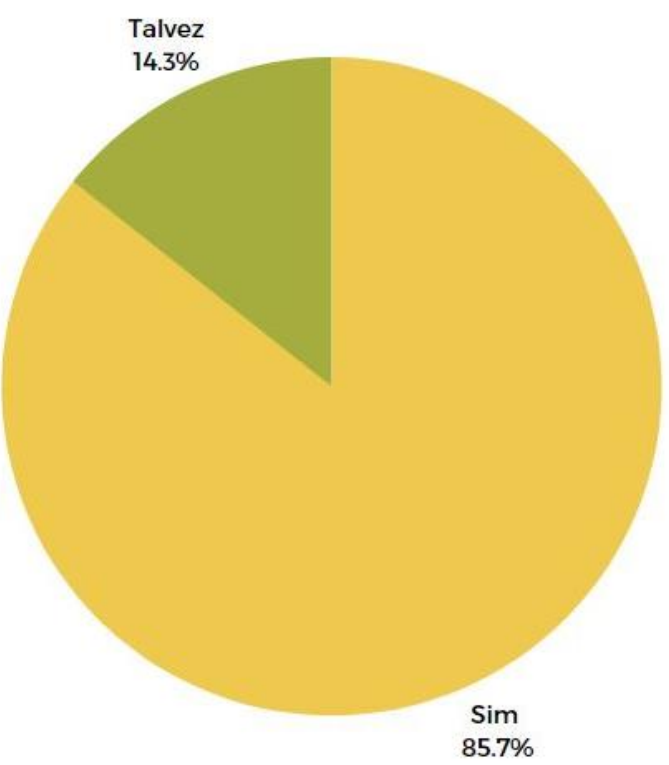

Fonte: Autoria própria.

\section{Promoção:}

\section{ABENGE}


Por fim, no que se trata de uma avaliação geral das capacitações, foi notado que $83,7 \%$ acharam excelente. Apesar de ser um resultado bastante positivo, cada vez mais procura-se melhorar, entretanto, percebe-se que o caminho é correto, pois muitos participantes estão satisfeitos com o que aprenderam e puderam aplicar os conhecimentos adquiridos em diversas formas.

\section{CONSIDERAÇÕES FINAIS}

Mediante os resultados supracitados, é notável a sua relevância, uma vez que tornou-se visível a importância de se possuir qualificação. Dessa forma, no que diz respeito à funcionalidade, percebe-se que as capacitações são necessárias para melhor formação do indivíduo no âmbito acadêmico e profissional, tendo em vista que carrega um conjunto de conhecimentos, e por isso, são prioridades no Capítulo Estudantil IEEE PES UFCG.

$\mathrm{O}$ desenvolvimento das capacitações permitiu reforçar fundamentos e aprendizados em áreas reconhecíveis - principalmente - para o curso de Engenharia Elétrica. Na qual foi perceptível que, não somente favoreceu os participantes, mas também os ministrantes, que ganharam conhecimentos e formação técnica.

Em virtude das capacitações serem realizadas também para os alunos ingressantes, além do conhecimento adquirido, ainda serve como um estímulo para manter os alunos interessados no curso, e consequentemente, minimizar a evasão.

Como notado nos resultados que cerca de $83,7 \%$ dos participantes consideraram as capacitações excelentes, percebe-se o quão é aplicável para o desempenho dos alunos nas disciplinas, em projetos ou até mesmo para uso pessoal. Ademais, tendo em vista que $71,4 \%$ dos partícipes destacaram que a aplicabilidade dos conteúdos adquiridos foi excelente, compactua-se que essas capacitações ofertadas recentemente podem servir de base para outras novas, pois está nítido que as capacitações acrescentam descomedidamente na formação pessoal, acadêmica e profissional.

Com relação às perspectivas futuras, propõe-se oferecer mais capacitações; apresentar novas versões do material disponibilizado; além de elevar o nível do conteúdo, a fim de propiciar um conhecimento mais completo e que possibilite uma aplicabilidade geral, isto é, tanto na área acadêmica e profissional, como para uso de exclusividade pessoal.

\section{Agradecimentos}

Agradecemos a Power and Energy Society pelo fornecimento dos kits educacionais de energias renováveis. A Universidade Federal de Campina Grande pelo suporte e pela estrutura disponibilizada. Por fim, a todas as escolas que nos receberam para aplicação do nosso projeto. 


\section{REFERENCIAS}

Artigos de periódicos:

OLIVEIRA, V. F. Trajetória e estado da arte da formação em Engenharia, Arquitetura e Agronomia. Formas Consultoria e Editoração Ltda, Brasília (DF), v. 1, 2010.

TONTINI, G.; WALTER, S. A. Pode-se identificar a propensão e reduzir a evasão de alunos? Ações estratégicas e resultados táticos para instituições de Ensino Superior. Avaliação, Campinas, Sorocaba (SP), v. 19, n. 1, p. 89-110, mar. 2014.

SILVA FILHO, R. L. L. et al. A evasão no ensino superior brasileiro. Cadernos de Pesquisa, São Paulo, v. 37, n. 132 p. 641-659, 2007.

\section{THE PERFORMANCE OF TRAINING IN THE ACADEMIC/PROFESSIONAL SCENARIO FOR UNDERGRADUATE STUDENTS AT THE FEDERAL UNIVERSITY OF CAMPINA GRANDE}

Abstract: From an educational perspective, dropout in higher education is considered a socioeducational problem, since it generates negative impacts on universities. It is noticeable that the evasion rate is increasing more and more, so, researchers have been intensifying their studies in search of improvements in this situation. After all, the job market needs professionals, mainly trained. With this in mind, the Student Chapter PES (Power and Energy Society) of the Federal University of Campina Grande (UFCG), belonging to the Institute of Electrical and Electronic Engineers (IEEE), performs training in Electrical Installations, Renewable Energies, Computer Formatting and Photoshop. Consequently, these qualifications were able to contribute vehemently to engagement, personal and professional training, and the interest for students to remain in the electrical engineering course.

Keywords: Education, Training, Engineering, IEEE, PES. 